\title{
Advanced Processing of Images Obtained from Wide-field Astronomical Optical Systems
}

\author{
M. Řřrábek, P. Páta
}

\begin{abstract}
The principal aim of this paper is to present a general view of the special optical systems used for acquiring astronomical image data, commonly referred to as WFC or UWFC (Ultra Wide Field Camera), and of their transfer characteristics. UWFC image data analysis is very difficult in general, not only because the systems have so-called space variant (SV) properties. Images obtained from UWFC systems are usually incorrectly presented due to a wide range of optical aberrations and distortions. The influence of the optical aberrations increases towards the margins of the field of view. These aberrations distort the point spread function of the optical system and rapidly cut the accuracy of the measurements. This paper deals with simulation and modelling of the UWFC optical systems used in astronomy and their transfer characteristics.
\end{abstract}

Keywords: PSF, UWFC, Zernike polynomial, aberration, space variant, deconvolution algorithm.

\section{Introduction}

The properties of UWFC astronomical systems along with specific visual data in astronomical images contribute to complicated evaluation of acquired image data. These systems contain many different kinds of optical aberrations, which have a negative impact on the image quality and imaging system transfer characteristics. Therefore, for precise astronomical measurements (astrometry, photometry) over the entire field of view (FOV), it is very important to comprehend how the optical aberrations affect the transfer characteristics of the optical system. Another question that arises is how the astronomical measurements depend on optical aberrations. A definition of the accurate point spread function (PSF) at any point in the FOV of the optical system could help us to restore the original image. Optical aberrations models for linear space invariant and variant (LSI/LSV) systems are therefore outlined in this paper. These models based on Zernike polynomials serve us as suitable tools for understanding how to estimate and fit the wavefront aberration of a real optical system, and give us an idea of intrinsic PSF. When the model of the PSF of a real acquisition system is known, we can use it for restoring the original image or for a precise evaluation of the astronomical measurements. Two experiments are presented in this paper. The first describes PSF model retrieval when we have original image data. The second focuses on using the PSF model in known deconvolution algorithms with which we can restore the original image.

\section{Astronomical image data processing and acquisition}

Most astronomical image data is provided by automatic robotic astronomical systems. The main idea of these automatic astronomical optical systems is based on long-term image data collection. The data is acquired with specific characteristics. These include especially focal length, spectral bands and sensor parameters. According to the focal length that is used, we can distinguish image data acquired in the primary focus of a big telescope (deep sky), a wide-field lens or a "fish-eye" lens for all sky data imaging. Astronomical image data contains specific visual data. An astronomical image usually consists of the dark background of the starry sky, together with bright points and clumps, which represent stars and galaxies. Image data can be divided into four groups [7]: Flat Field, Dark Frame, Light Image and Deep Sky Light Image. Before processing and evaluating astronomical images, it is necessary to make corrections of these images. Dark frame compensation and flat field compensation are two of the most frequently used pre-processing methods.

Real data from double-station video observation of meteors [10,11] is used for our simulations and modelling. UWFC image data analysis is very difficult in general. There are many different kinds of optical aberrations and distortions in these systems. Moreover, the objects in ultra wide-field images are very small (a few pixels per object dimension). The influence of the optical aberrations increases most to- 


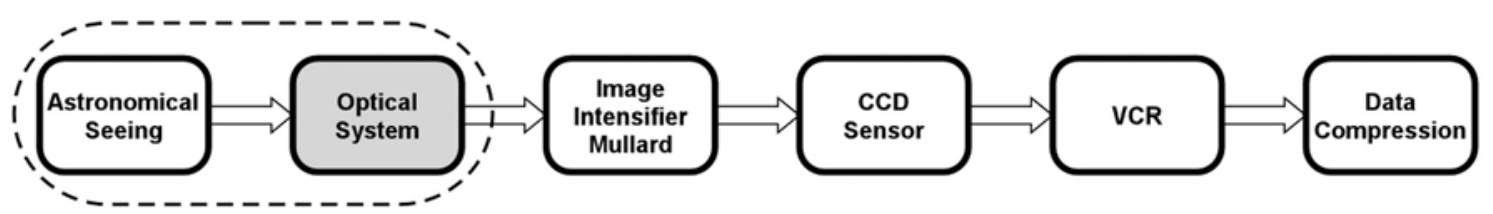

Fig. 1: Block diagram of image processing in a video observation system

wards the margins of the FOV (high angular distance from the optical axis of the system). These aberrations distort the PSF of the optical system and rapidly cut the accuracy of the measurements. The optical aberrations are dependent on spatial data, which affects the transfer characteristics of optical systems and makes them spatially variant.

\section{$3 \quad$ Stellar object profile}

There are two common functions for fitting the profiles of stars - Gaussian and Moffat [7,11]. The aim is to match a star's profile as well as possible with the Gaussian or Moffat profile, and then to store the parameters of the fit. If the star is ideal, it will be represented by a small "dot" — PSF (Point Spread Function). Unfortunately, because of many different distortions (see below), the dot is "blurred" all around, and the star's profile is close to the Gaussian function. The more it is blurred the worse is the PSF of the whole imaging system. If the system is linear and space-invariant, the PSF of all stars will be the same. The centre of a star usually has a profile closer to the Gaussian function, while the more distant parts of a star are closer to the Moffat profile. Hence the ideal fitting function combines Gaussian and Moffat profiles. It is obvious that the centre of the star is fitted very well, whereas the more distant parts are fitted only poorly. Figure 1 presents a simplified block diagram of various factors affecting the final image and stellar profile. We assume the first two blocks for our purposes; however, we consider that these blocks are placed in a black box. In our experiments, we do not take into account a model of atmospheric turbulence. Other blocks implicate disruptive effects, which bring unwanted artefacts in PSF. One of the most difficult effects is brought on by recording and compression. In order to improve the subjective image quality, the video tape recording and even JPEG compression use a sharpening mechanism which results in rapid transients at the edge of PSF. These parts of the electro-optics transfer system are not considered in our simulations and modelling.

\section{Optical aberration modelling}

Optical aberration can be described using a so-called wave aberration function. The wave aberration $[1,5]$ function is defined as the distance (in optical path length) from the reference sphere to the wavefront in the exit pupil measured along the ray. Zernike polynomials are used for describing high order wavefront aberrations with high precision. Zernike polynomials are normally expressed in polar coordinates $(\rho, \theta)$ in the exit pupil, where $0 \leq \rho \leq 1,0 \leq$ $\theta \leq 2 \pi$. A Zernike polynomial consists of two factors, the normalization factor $N_{n}^{m}$ and the radial polynomial $R_{n}^{|m|}(\rho)$. Zernike polynomials are defined as $[1,4]$ :

$Z_{n}^{m}(\rho, \theta)=\left\{\begin{array}{l}N_{n}^{m} R_{n}^{|m|}(\rho) \cos (m \theta) \\ \text { for } m \geq 0,0 \leq \rho \leq 1,0 \leq \theta \leq 2 \pi \\ -N_{n}^{m} R_{n}^{|m|}(\rho) \sin (m \theta) \\ \text { for }, m<0,0 \leq \rho \leq 1,0 \leq \theta \leq 2 \pi,\end{array}\right.$

for a given $n$, the number $m$ can take values of $-n,-n+2,-n+4, \ldots, n$. The normalization factor $N_{n}^{m}$ and the radial polynomial $R_{n}^{|m|}(\rho)$ can be expressed as

$$
\begin{aligned}
& N_{n}^{m}=\sqrt{\frac{2(n+1)}{1+\delta_{m 0}}}, \\
& R_{n}^{|m|}(\rho)= \\
& \sum_{s=0}^{\frac{n-|m|}{2}} \frac{(-1)^{S}(n-s) !}{s ![0,5(n+|m|)-s] ![0,5(n-|m|)-s] !} \rho^{n-2 s} .
\end{aligned}
$$

where $\delta_{m 0}=1$ for $m=0, \delta_{m 0}=0$ for $m \neq 0$. The wavefront aberration function may be expressed by Zernike polynomials $[1,5,7]$ as

$$
\begin{aligned}
W(\rho, \theta)= & \sum_{n}^{k} \sum_{m=-n}^{n} W_{n}^{m} Z_{n}^{m}(\rho, \theta)= \\
& \sum_{n}^{k}\left\{\sum_{m=-n}^{-1} W_{n}^{m}\left(-N_{n}^{m} R_{n}^{|m|}(\rho) \sin (m \theta)\right)+\right. \\
& \left.\sum_{m=0}^{n} W_{n}^{m}\left(N_{n}^{m} R_{n}^{|m|}(\rho) \cos (m \theta)\right)\right\}
\end{aligned}
$$

where $k$ is the polynomial order of the expansion, and $W_{n}^{m}$ is the coefficient of the $Z_{n}^{m}$ mode in the expansion, i.e. it is equal to the RMS wavefront error for that mode. 


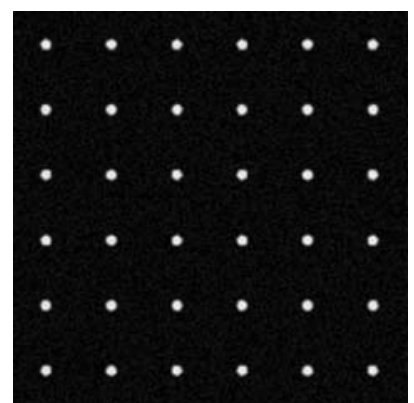

a)

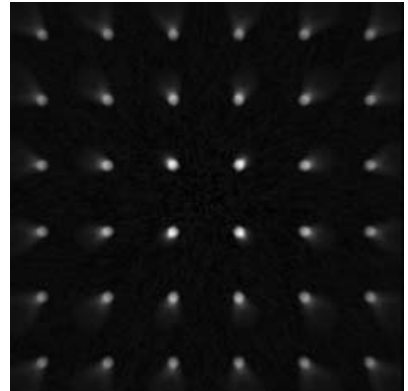

b)

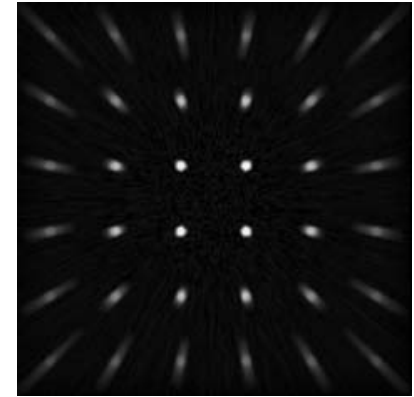

c)

Fig. 2: Visualization of coma and astigmatism. a) The testing image. b) SV image distorted by coma. c) SV image distorted by astigmatism

\section{$5 \quad$ Effect of wavefront aberration on the PSF}

Optical systems with all aberrations compensated are called diffraction limited. The influences of aberrations on image quality can be expressed as the wavefront error at the exit pupil, and their effects on the transfer characteristics can be expressed as the change in the PSF size and shape. Changes in the size and shape of PSF blur the image. When an imaging system is diffraction limited, the PSF consists of the Fraunhofer diffraction pattern of the exit pupil [2]. The relation between the object and the image of a space invariant optical system can be expressed by the convolution in the spatial domain (the object irradiance distribution is convolved with the impulse response) [2]. The PSF of the LSI optical imaging system can be expressed as

$$
\begin{aligned}
& \operatorname{PSF}(u, v, \delta, \varphi)= \\
& \left|F T\left\{p(x, y) \exp \left(-\mathrm{i} \frac{2 \pi}{\lambda} W(\rho, \theta, \delta, \varphi)\right)\right\}\right|^{2},
\end{aligned}
$$

where $p(x, y)$ defines the shape, size and transmission of the exit pupil, $\exp [(-\mathrm{i} 2 \pi / \lambda) W(\rho, \theta, \delta, \phi)]$ accounts for the phase deviation of the wavefront from a reference sphere, and $(\delta, \varphi)$ are the polar coordinates at the object plane - these coordinates equal $(0,0)$ all the time if we consider an LSI optical system.

If we assume the spatial variant system with anisotropic PSF, then the aberration wavefront is different for each source point, see Figure 2. The real image is always obtained from the spatially variant optical system. An example of an image acquired from a real SV optical system is shown in Figure 3a, where can we also see the influences of aberrations in the middle of the image and at the edge. The PSF of this optical system is different in the middle of the image and at the edge, due to optical aberrations. The differences of the PSFs in the two positions are compared in Figure $3 \mathrm{~b}$ and Figure 3c.

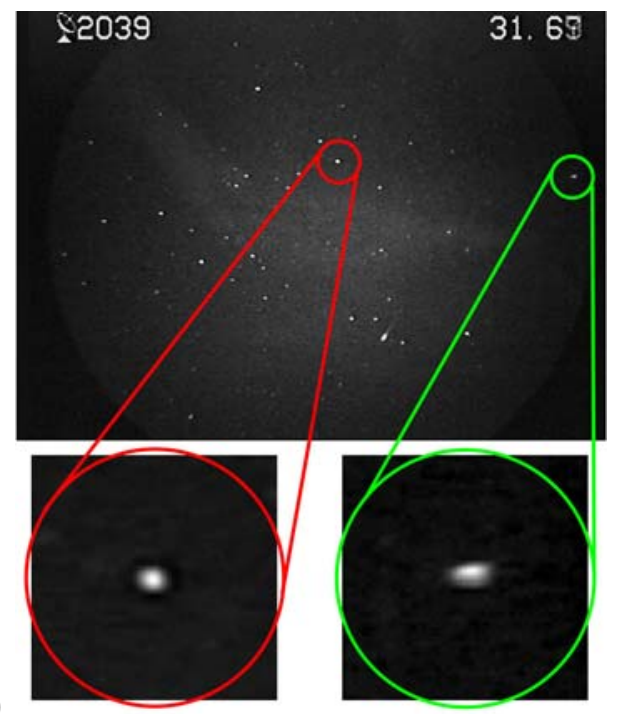

a)

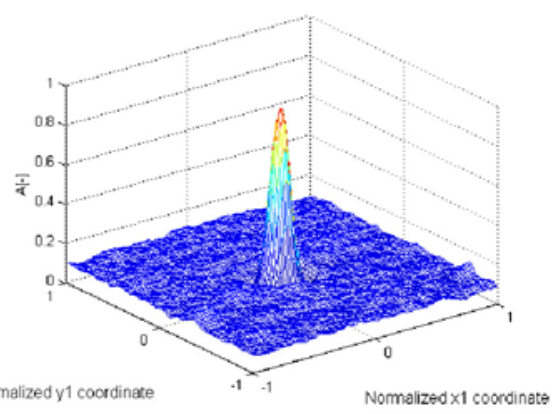

b)

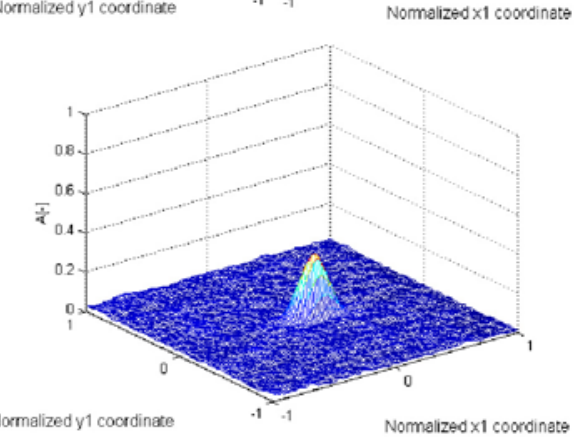

Fig. 3: a) Input image data from the system at the Ondřejov base. b) The object profile near the optical axes. c) Object profile on the edge of FOV 


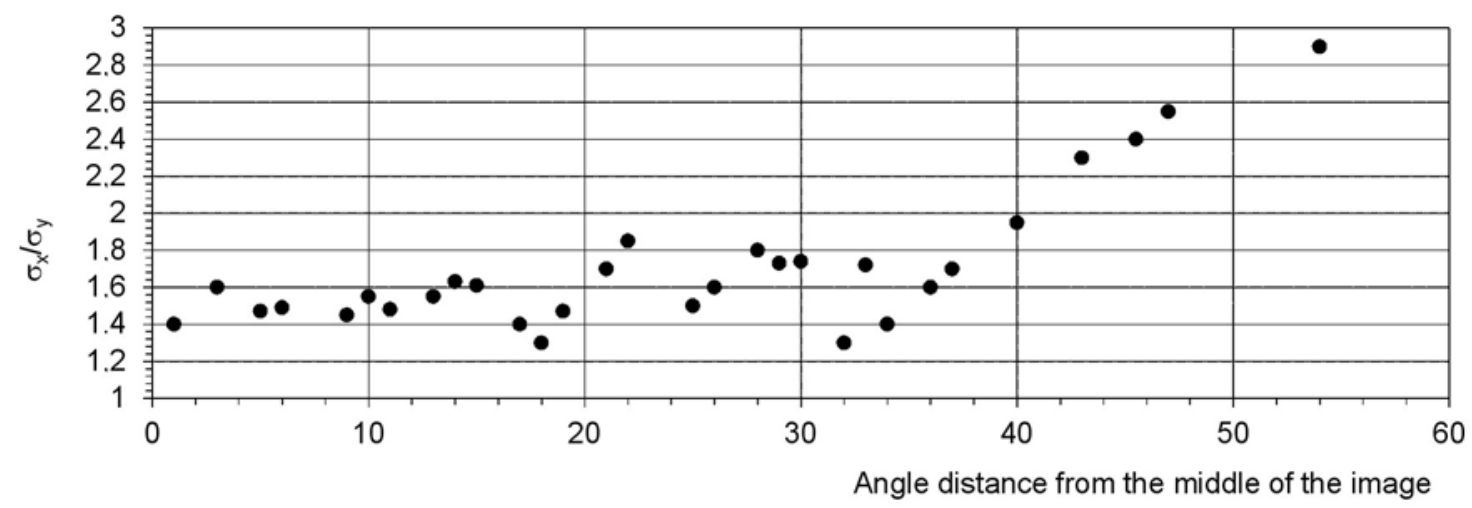

Fig. 4: Ellipticity (an ideal PSF deformation) of the profiles of stars as a function of the angle distance from the middle of an all-sky image

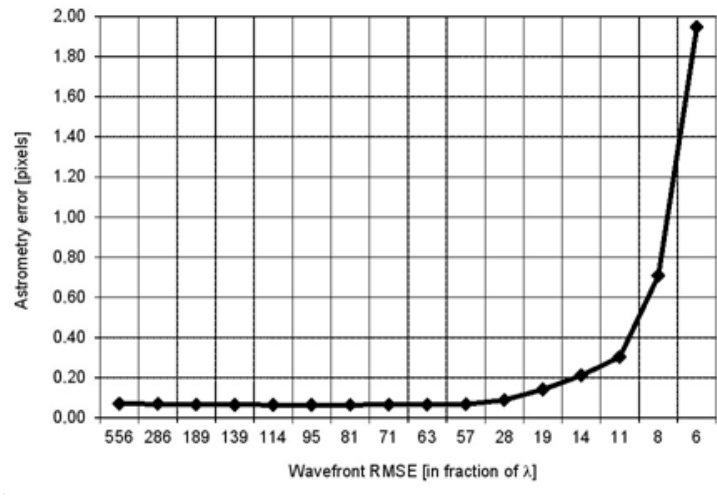

a)

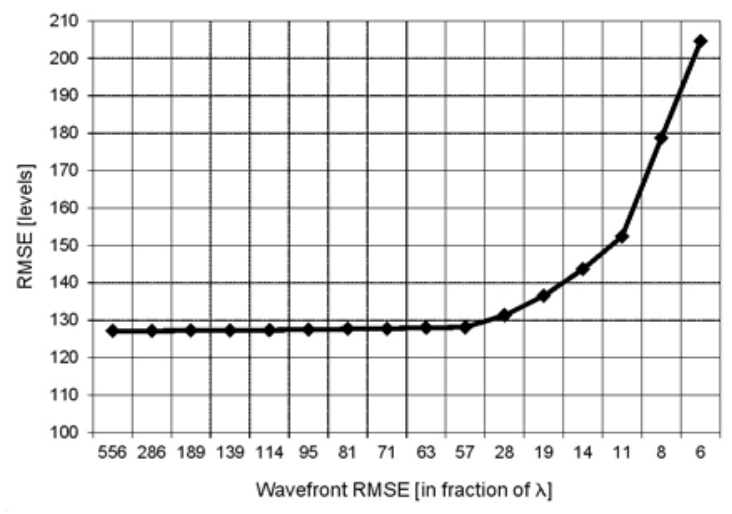

b)

Fig. 5: a) Dependence of the astrometric error (in pixels) on wavefront aberration error (in fraction of $\lambda$ ), b) RMS error (in levels) versus wavefront aberration error (in fraction of $\lambda$ )

\section{Effect of Wavefront Aberration on Astrometric Measurements}

Unlike aperture photometry, precise astrometry demands high quality images. Even slight distortion may cause inaccurate determination of the position or movement of a stellar object. The error depends on the magnitude of the measured star - the greater the magnitude, the greater the error can be. Even a quite small compression rate may cause a wrong detection, especially when considering faint stars.

If the system is space-variant, the PSF of the stars in an image differs. The profiles of stars are not circular but rather elliptical, especially in the case of inferior lenses and greater distance from the middle of the image. The ellipticity is qualified by $\sigma_{x}$ and $\sigma_{y}$ (while considering the Gaussian function), where $\sigma_{x}$ and $\sigma_{y}$ are the distance from the centre of the Gaussian at which the Gaussian is equal to $\exp (-0,5)$ of its central value. $\sigma_{x}$ and $\sigma_{y}$ are the major and minor half-axis of the ellipse, perpendicular to each other. The ellipticity in the radial direction can grow significantly with the angle distance from the middle of the image, especially for wide-angle images, see Figure 4.
All of the measurements in this section were done in IRAF (Image Reduction and Analysis Facility) software [13]. This software system enables the user to measure, reduce and analyse astronomical data and write her/his own scripts. The program is "open source". The reader can find further information at http://iraf.noao.edu. SkyCat can be used for visualization of images and for access to catalogues, for example http://archive.eso.org/skycat.

The effect of coma wavefront aberration on the precision of astrometric measurements (e.g. position of objects) is demonstrated in Figure 5a. We can see that the coma aberration has no influence on the precision of astrometric measurements for values of wavefront aberration error less than $\lambda / 50$. The measurement error grows with the wavefront aberration error, and for wavefront aberrations error values greater than $\lambda / 10$ it is no longer acceptable. The second graph represents the effect of coma wavefront aberration on the RMSE of a distorted image in signal levels, see Figure 5b. This effect is insignificant for wavefront aberration error values less than $\lambda / 100$. RMSE further increases with wavefront aberration error. For wavefront aberration error greater than $\lambda / 10$, the increase in RMSE values is over $30 \%$, and is also no longer acceptable. We assume only the 
a)

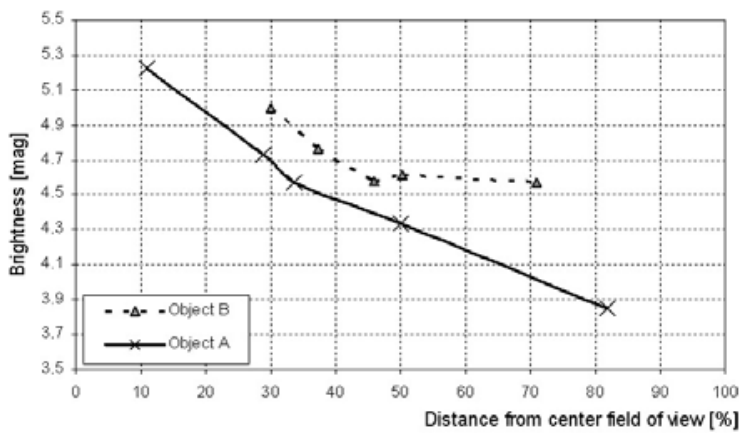

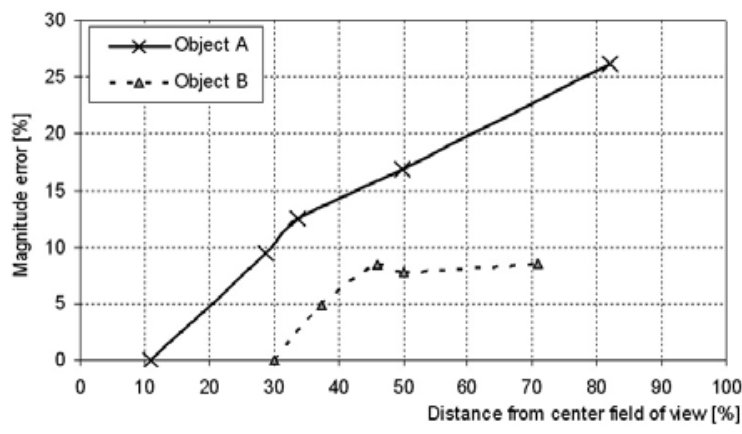

b)

Fig. 6: a) Dependence of the brightness stars on distance from centre of FOV, b) Dependence of the magnitude error of stars on the distance from centre of FOV

coma aberration included in the optical shift invariant system.

\section{Experiments and Results}

\subsection{Estimation of aberration}

If we want to use the deconvolution algorithm for precise restoration of the SV UWFC system, we need to know what the PSF looks like. One approach is to find the PSF of the optical system from empirical image data. Here we describe the procedure for obtaining the model of PSF from real image data.

Our experiments involve analysing real image data and comparing it with the modelled image data. Real data from the double-station video observation project is used for our experiments. The stars vary not only their own position, but also their brightness and shape. The space variant properties are represented in Figures 6a) and 6b), which show how the magnitude and the magnitude error vary in dependence on distance from the centre of FOV. In principle, the brightness of stars has a decreasing character.

Within the double-station video observation project we obtain a sequence in non-compressed AVI format from the observation system. A star which changes its position from the edge to the middle of the image, or vice versa, is taken as a suitable for our purposes. At first, several frames from each sequence form the uncorrected testing image, which is determined as the median of the used frames. In the next step, we pre-process the image using flat field and dark frame correction. We can also use noise and background removal. The next step is to iso-

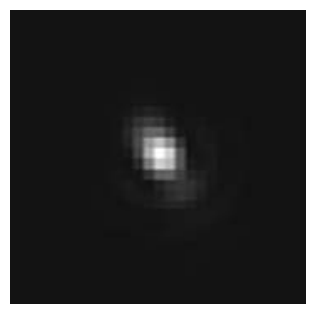

a)

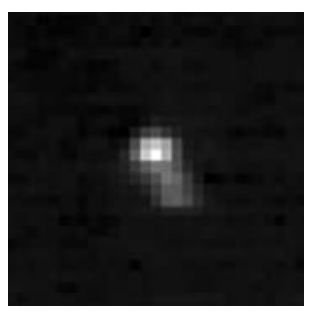

b)

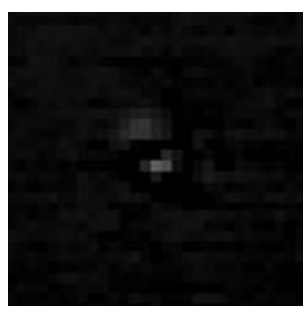

c) late the star at discrete times when it changes its own position from the middle of FOV to the edge of FOV. We therefore cut the stars as sub-images with 15 by 15 pixel proportions. Then the comparison of the real stellar profile and the model profile is implemented [12]. A generalized block diagram of the algorithm that estimates the optical aberrations of the real system is shown in Figure 7. The input parameters are the wavefront aberration (i.e., the Zernike coefficients) and PSF computed according equation (4). Then the real object profile is compared with the suggested model - both of them have the same FWHM [7]. The suitability of the resulting PSF for the model is evaluated on the basis of the RMS error between the modelled PSF and the real object profile. The result for the chosen star is presented in Figure 8. As can be seen, this model assumes only two varying aberrations (coma and astigmatism) with a constant defocus value.

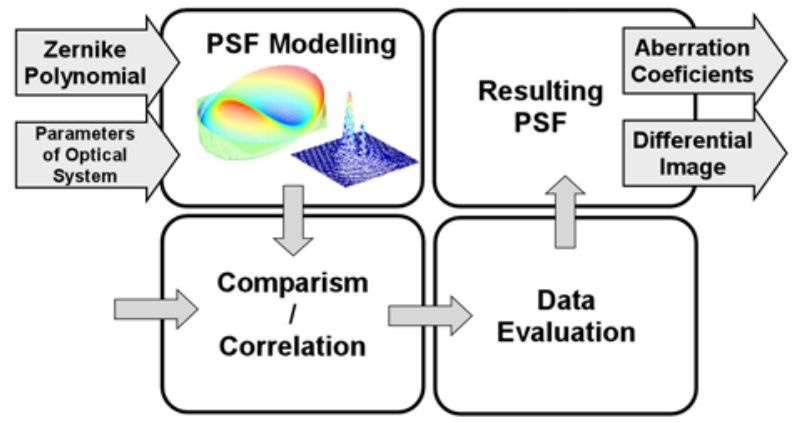

Fig. 7: Generalized block diagram of an estimation algorithm

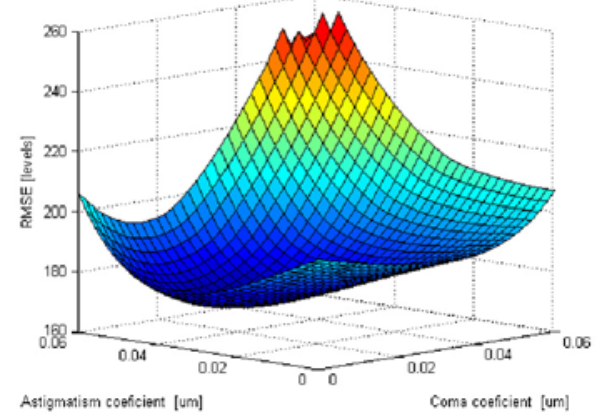

d)

Fig. 8: Results of estimation. a) Model of the star profile, b) Original of the chosen star, c) Differential image for the estimated and real star profile, d) Dependence of the RMS error profile on coma and astigmatism wavefront aberration error 


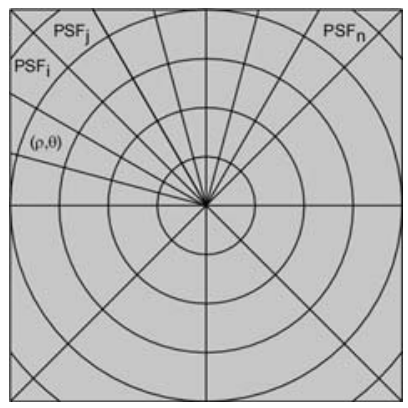

a)

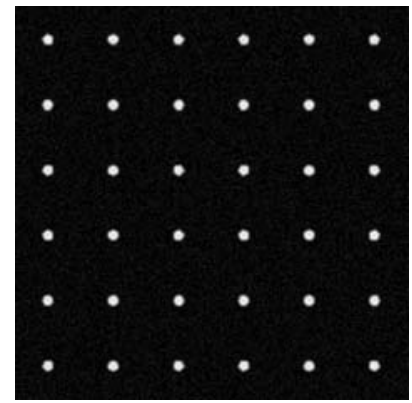

b)

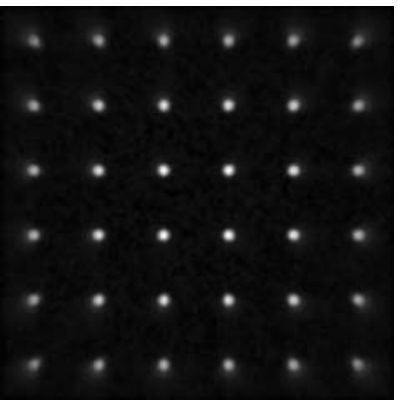

c)

Fig. 9: a) Model of the PSI optical system, b) Source testing image, c) Image passed through the SV optical system with coma aberration only

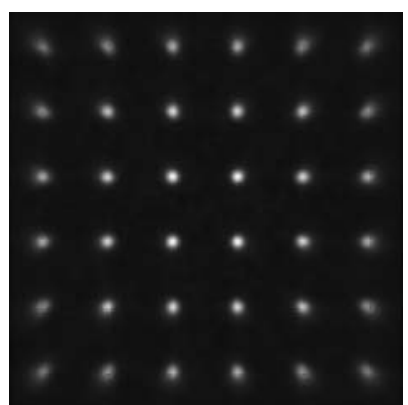

a)

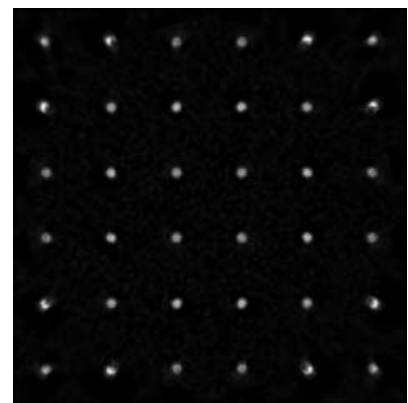

b)

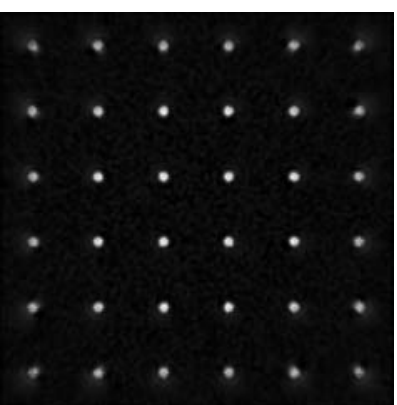

c)

Fig. 10: Image restored using the Wiener deconvolution algorithm, b) Deconvolution using the Lucy — Richardson algorithm, c) Deconvolution using the maximum likelihood algorithm

\subsection{Model of spatially variant deconvolution}

The principal difficulty in spatially variant (SV) systems is that the Fourier approach can no longer be used for restoring [6] the original image. If we want to use the Fourier method for the deconvolution process, we need to split the original image. The transfer characteristics of each part are described by unique PSF. This system is referred to as a partially space invariant system [8] and we use it in our experiments. Such a system has parametric PSF — for each value of a parameter (in our case it is the coordinate of the object at the object plane) PSF takes a different size and shape according to the aberrations that are contained. Thus we obtain a number of PSFs, one for each region referred to as an isoplanatic patch [8], within which the system is approximately invariant. To describe the imaging system fully, the impulse response appropriate for each isoplanatic patch should be specified.

The wavefront aberration function for the LSV optical system can be described as

$$
W(\rho, \theta, \delta, \varphi)=\sum_{n}^{k} \sum_{m=-n}^{n} W_{n}^{m}(\delta, \varphi) Z_{n}^{m}(\rho, \varphi-\theta),
$$

where $W_{n}^{m}(\delta, \varphi)$ is the RMS wavefront error for aber- ration mode $m, n$ and for object polar coordinate $(\delta, \varphi)$. Using the partially space invariant system allows us to describe the transfer characteristics in individual patches by Fourier approaches, according to equation (4). Thus we obtain a number of space invariant PSFs, one for each isoplanatic patch. Now we can also use the Fourier approach for image deconvolution. The optical system is divided into 40 isoplanatic patches in our experiments, see Figure 9. Three deconvolution algorithms [3, 4] — the Wiener, LucyRichardson and maximum likelihood algorithm are used for restoring the original image, see Figure 10 .

\section{Conclusions}

This paper has discussed ways of improving astronomical measurements. The first approach is to use the novel star profile model for restoring the original image, which is undistorted and is suitable for precise astrometry using ordinary fitting models (Gaussian, Moffat). The second approach for improving astronomical measurements is to use a different evaluation and detection algorithm. This involves creating a new PSF fitting model based, e.g., on the Zernike polynomial and using it for detection and astrometric measurements. The situation is complicated by fact that all wide field astronomical observational systems 
are SV. Therefore, the shape of a stellar object varies across the entire FOV, and Gaussian or Moffat fitting procedures are useless for precise astrometric measurements, especially towards the margins of FOV.

An experiment for estimating the optical aberrations of real optical systems was implemented. A comparison of the model PSF and the real object profile was performed using the RMS error. Obviously the model should have more input parameters, especially more varying optical aberrations. SV deconvolution was addressed, and a model of the partially space variant optical system was implemented. Only the "brute force" method was used for restoring the test images. The results of various deconvolution algorithms were demonstrated.

\section{Acknowledgement}

This work has been supported by grants no. GA205/09/1302 "Study of sporadic meteors and weak meteor showers using automatic video intensifiers cameras", and GA P102/10/1320 "Research and modelling of advanced methods of image quality evaluation" of the Grant Agency of the Czech Republic.

\section{References}

[1] Born, M., Wolf, E.: Principles of Optics. 2nd ed. London, Pergamon Press, 1993.

[2] Goodman, J. W.: Introduction to Fourier Optics. Boston, McGraw-Hill, 1996.

[3] Campisi, P., Egiazarian, K.: Blind Image Deconvolution: Theory and Applications. CRC, 2007.

[4] Janson, P. A.: Deconvolution of Images and Spectra. $2^{\text {nd }}$ edition. Academic Press, London, 1997.

[5] Welford, W. T.: Aberration of the Symmetrical Optical System. Academic Press, London, 1974.
[6] Shannon, R. R., Wyant, J. C.: Applied Optics and Optical Engineering. Academic Press, Lodon, 1992.

[7] Starck, J., Murtagh, F.: Astronomical Image and Data Analysis. Springer Verlag, Berlin, 2002.

[8] Trussel, H. J., Hunt, B. R.: IEEE Trans. Acoustic Speech Sig. Proc., 608, 157, 1978.

[9] Maeda, P. Y.: Zernike Polynomials and their Use in Describing the Wavefront Aberrations of the Human Eye. 2003.

[10] Hudec, R., Spurny, M., Krizek, P., Páta, P., Řř́ábek, M.: Low-Cost Optical All-Sky Monitor for Detection of Bright OTs of GRBs In: Gamma-Ray Burst: Sixth Huntsville Symposium. Austin: American Institute of Physics, 2009, p. 215-217. ISBN 978-0-7354-0670-4.

[11] Řeřábek, M., Páta, P., Koten, P.: Processing of the Astronomical Image Data obtained from UWFC Optical Systems. In Proceedings of SPIE - 7076 - Image Reconstruction from Incomplete Data $V$. Washington: SPIE, 2008. ISBN 978-0-8194-7296-0.

[12] Řrerábek, M., Páta, P.: Advanced processing of astronomical images obtained from optical systems with ultra wide field of view In: Applications of Digital Image Processing XXXII. Bellingham (Washington State): SPIE, 2009, p. 1-4. ISBN 978-0-8194-7733-0.

[13] IRAF - Image Reduction and Analysis Facility, http://iraf.noao.edu/, 2007.

M. Řeřábek

P. Páta

E-mail: rerabem@.fel.cvut.cz

Czech Technical University in Prague

Faculty of Electrical Engineering

Department of Radio Engineering

Technická 2, 16627 Prague 6, Czech Republic 